\title{
Nodal solutions of an NLS equation concentrating on lower dimensional spheres
}

\author{
Giovany M Figueiredo ${ }^{1}$ and Marcos TO Pimenta ${ }^{2 *}$
}

\author{
"Correspondence: \\ pimenta@fct.unesp.br \\ ${ }^{2}$ Departamento de Matemática e \\ Computação, Faculdade de Ciências \\ e Tecnologia, Universidade Estadual \\ Paulista - Unesp, Presidente \\ Prudente, SP 19060-900, Brazil \\ Full list of author information is \\ available at the end of the article
}

\begin{abstract}
In this work we deal with the following nonlinear Schrödinger equation:

$$
\left\{\begin{array}{l}
-\epsilon^{2} \Delta u+V(x) u=f(u) \quad \text { in } \mathbb{R}^{N} \\
u \in H^{1}\left(\mathbb{R}^{N}\right)
\end{array}\right.
$$

where $N \geq 3, f$ is a subcritical power-type nonlinearity and $V$ is a positive potential satisfying a local condition. We prove the existence and concentration of nodal solutions which concentrate around a $k$-dimensional sphere of $\mathbb{R}^{N}$, where $1 \leq k \leq N-1$, as $\epsilon \rightarrow 0$. The radius of such a sphere is related with the local minimum of a function which takes into account the potential $V$. Variational methods are used together with the penalization technique in order to overcome the lack of compactness.
\end{abstract}

MSC: $35 J 60 ; 35 J 10 ; 35 J 20$

Keywords: variational methods; nodal solutions; concentration on manifolds

\section{Introduction}

In the last decades, motivated by the great interest that this problem attracts in quantum mechanics, many researchers have dedicated their efforts to the study of the nonlinear Schrödinger equation

$$
i \hbar \frac{\partial \psi}{\partial t}=-\frac{\hbar^{2}}{2 m} \Delta \psi+W(x) \psi-|\psi|^{p-1} \psi, \quad(t, x) \in \mathbb{R} \times \mathbb{R}^{N}
$$

Of particular interest are the so-called standing wave solutions which consist in solutions with a particle-like behavior. It is obtained by the $A n s a t z \psi(t, x)=e^{-i E t / \hbar} u(x)$ which associates the NLS equation to its stationary version

$$
-\epsilon^{2} \Delta u+V(x) u=|u|^{p-1} u \quad \text { in } \mathbb{R}^{N}
$$

where $\epsilon^{2}=\hbar^{2} / 2 m$ and $V(x)=W(x)-E$. As far as (1.1) is concerned, the behavior of the solutions when $\epsilon \rightarrow 0$ has a great physical interest since it describes the transition from quantum to classical mechanics, being called semiclassical states. On this specific subject, many authors have worked on spike-layered solutions which are nontrivial ground-state

(c) 2015 Figueiredo and Pimenta. This article is distributed under the terms of the Creative Commons Attribution 4.0 International License (http://creativecommons.org/licenses/by/4.0/), which permits unrestricted use, distribution, and reproduction in any medium, provided you give appropriate credit to the original author(s) and the source, provide a link to the Creative Commons license, and indicate if changes were made. 
points of the associated energy functional and tend to concentrate around one or more critical points of the potential $V$. We could cite some quite influential works on this subject, as the pioneering work of Floer and Weinstein [1], which have inspired the works of Rabinowitz [2], Wang [3], Del Pino and Felmer [4], which have influenced so many other works in the last three decades.

In the last ten years, solutions which concentrate on higher dimensional sets have received more and more attention. The first work which seems to show this kind of result is [5] in which the authors study an NLS equation on a bounded domain with Neumann boundary condition and prove the existence of a sequence of solutions which concentrate on some component of the boundary. One of the first works dealing with solutions concentrating around a sphere is [6] in which Ambrosetti, Malchiodi and Ni give necessary and sufficient conditions under which (1.1) exhibits solutions concentrating around a sphere. The radius of such a sphere is given by a minimum point of a function $\mathcal{M}$, which takes into account the value of the radial potential $V(|x|)$. The role played by $\mathcal{M}$ is in order to balance the potential energy (coming from $V$ ) and the volume energy which arise from the other terms of the energy functional (see the introduction of [6] for more details). In fact, sphere-concentrating solutions show a rather different behavior when compared with spike-layered ones. To be more specific, in [6], the authors prove the existence of sphereconcentrating solutions to (1.1) even for critical or supercritical exponent $p$. This is in a strike contrast with the fact that, as showed in [7], no spike-layered solution exists to (1.1) for $p=2^{*}-1$. Other significant difference is that the energy of the sphere-concentrating solutions tends to zero in contrast with that of spike-layered solutions which converges to the mountain-pass level of the energy functional. In these and so many other works ([8-11] for example), Lyapunov-Schmidt reduction methods have been used in order to construct the sphere-concentrating solutions for Schrödinger equations, Schrödinger-Poisson systems and other related problems.

More recently, in [12] Bonheure et al. proved the existence of solutions concentrating around a $k$-dimensional sphere of $\mathbb{R}^{N}$ for all $k \in\{1, \ldots, N-1\}$ to the following equation:

$$
-\epsilon^{2} \Delta u+V(x) u=K(x) f(u) \text { in } \mathbb{R}^{N},
$$

where the potentials $V$ and $K$ satisfy rather generic conditions, allowing $V$ even to vanish on the infinity. To do so, they use a modification of the penalization technique, originally presented in [4], in such a way that compactness is recovered to the modified energy functional. Because of the generality of conditions to which $V$ and $K$ are subjected, in order to prove that the solutions of the modified problem are solutions of the original one, they made a thorough analysis with some barrier functions which bound the solutions from above. In [13] the authors employ a similar argument in order to show the existence of solutions concentrating on circumferences of $\mathbb{R}^{3}$, to a Schrödinger-Poisson system.

In the spike-layered solutions setting, the existence of sign-changing (or nodal) solutions was investigated by some authors. In [14] and [15], Alves and Soares study problem (1.2), with $K$ to be a constant, and prove the existence of nodal solutions which concentrate on minima of the potential $V$. They consider $f$ as a subcritical power-type nonlinearity in their first work and as presenting a critical exponential growth at infinity in the second. In both they employ the penalization technique together with a careful analysis of the profile 
of the solutions. In [16], Sato proposes a different kind of penalization in order to show the existence of multi-peak nodal solutions to a Schrödinger equation with a vanishing potential.

A question that naturally arises is whether there exists a sequence of nodal solutions to the NLS equation which concentrate around a $k$-dimensional sphere. In this work we give a positive answer to this question. More specifically, we study the existence and concentration of nodal solutions to the following nonlinear Schrödinger equation:

$$
\left\{\begin{array}{l}
-\epsilon^{2} \Delta u+V(x) u=f(u) \quad \text { in } \mathbb{R}^{N} \\
u \in H^{1}\left(\mathbb{R}^{N}\right)
\end{array}\right.
$$

where $N \geq 3$, exhibiting a cylindrical symmetry which implies this sort of concentration. The nonlinearity $f$ is assumed to be a $C^{1}(\mathbb{R})$ odd function satisfying the following:

$\left(f_{1}\right)$ There exists $v>1$ such that $f(|s|)=o\left(|s|^{v}\right)$ as $s \rightarrow 0$;

$\left(f_{2}\right)$ There exist $c_{1}, c_{2}>0$ such that $\left|f^{\prime}(s)\right| \leq c_{1}+c_{2}|s|^{p-1}$, where $0<p<\frac{2 N}{N-2}-2$;

$\left(f_{3}\right)$ There exists $\theta>2$ such that

$$
0<\theta F(s) \leq f(s) s \quad \text { for } s \neq 0,
$$

where $F(s)=\int_{0}^{s} f(t) d t$

$\left(f_{4}\right) s \mapsto f(s) / s$ is increasing in $s>0$ and decreasing for $s<0$.

The potential $V$ will be assumed to satisfy a symmetry condition which we explain in the next section.

\subsection{Statement of the main result}

Let $1 \leq k \leq N-1$ be an integer which determines the dimension of the sphere in which the solutions obtained are going to concentrate. Consider $\mathcal{H}$ to be an $(N-k-1)$-dimensional linear subspace of $\mathbb{R}^{N}$ and note that $\mathcal{H}^{\perp}$ is a $(k+1)$-dimensional subspace. All along the paper we use the notation for $x \in \mathbb{R}^{N}$ as $x=\left(x^{\prime}, x^{\prime \prime}\right)$, in which $x^{\prime} \in \mathcal{H}, x^{\prime \prime} \in \mathcal{H}^{\perp}$ are such that $x=x^{\prime}+x^{\prime \prime}$.

From now on, if $h: \mathbb{R}^{N} \rightarrow \mathbb{R}$ is a function, by saying that $h\left(x^{\prime}, x^{\prime \prime}\right)=h\left(x^{\prime},\left|x^{\prime \prime}\right|\right)$ (which rigorously does not make sense), we mean that $h\left(x^{\prime}, y\right)=h\left(x^{\prime}, z\right)$ for all $y, z \in \mathcal{H}^{\perp}$ such that $|y|=|z|$.

The condition in $V$ which is considered is the following:

$\left(V_{1}\right)$ There exists $V_{0}>0$ such that $V_{0} \leq V(x)$ and, for all $x \in \mathbb{R}^{N}, V(x)=V\left(x^{\prime}, x^{\prime \prime}\right)=$ $V\left(x^{\prime},\left|x^{\prime \prime}\right|\right)$.

Unlike spike-layered solutions, whose concentration occurs around minimum points of $V$, the solutions we are going to study concentrate around minimum points of an auxiliary potential. To see how we define them, let us consider the limit problem

$$
-\Delta u+a u=f(u) \quad \text { in } \mathbb{R}^{N-k} .
$$


It is well known (see [2] for instance) that there exists a ground state solution $w \in H^{1}\left(\mathbb{R}^{N-k}\right)$ of (1.4) which minimizes the energy functional

$$
I_{a}(u)=\frac{1}{2} \int_{\mathbb{R}^{N-k}}\left(|\nabla u|^{2}+a u^{2}\right) d x-\int_{\mathbb{R}^{N-k}} F(u) d x,
$$

in the corresponding Nehari manifold given by

$$
\mathcal{N}_{a}=\left\{u \in H^{1}\left(\mathbb{R}^{N-k}\right) \backslash\{0\} ; I_{a}^{\prime}(u) u=0\right\}
$$

We define the ground-energy function $\mathcal{E}: \mathbb{R}^{+} \rightarrow \mathbb{R}^{+}$by

$$
\mathcal{E}(a)=\inf _{\mathcal{N}_{a}} I_{a}
$$

Finally, we define the auxiliary potential $\mathcal{M}: \mathbb{R}^{N} \rightarrow(0,+\infty]$ by

$$
\mathcal{M}(x)=\left|x^{\prime \prime}\right|^{k} \mathcal{E}(V(x))
$$

where $x=\left(x^{\prime}, x^{\prime \prime}\right), x^{\prime} \in \mathcal{H}$ and $x^{\prime \prime} \in \mathcal{H}^{\perp}$.

On the auxiliary potential $\mathcal{M}$, we impose the following condition:

$\left(\mathcal{M}_{1}\right)$ There exists an open bounded set $\Omega \subset \mathbb{R}^{N}$ such that if $\left(x^{\prime}, x^{\prime \prime}\right) \in \Omega$ then $\left(x^{\prime}, y^{\prime \prime}\right) \in \Omega$ for all $y^{\prime \prime} \in \mathcal{H}^{\perp},\left|x^{\prime \prime}\right|=\left|y^{\prime \prime}\right|$. Moreover,

$$
0<\mathcal{M}_{0}:=\inf _{x \in \Omega} \mathcal{M}(x)<\inf _{x \in \partial \Omega} \mathcal{M}(x)
$$

Before we state our main result, let us define, for $x=\left(x^{\prime}, x^{\prime \prime}\right), y=\left(y^{\prime}, y^{\prime \prime}\right) \in \mathbb{R}^{N}$,

$$
d_{k}(x, y)=\sqrt{\left(x^{\prime}-y^{\prime}\right)^{2}+\left(\left|x^{\prime \prime}\right|-\left|y^{\prime \prime}\right|\right)^{2}}
$$

as to be the distance between the $k$-dimensional spheres centered at the origin, parallel to $\mathcal{H}^{\perp}$ and of radius $\left|x^{\prime \prime}\right|$ and $\left|y^{\prime \prime}\right|$, respectively. Now we can finally state our main result.

Theorem 1.1 Letf satisfy $\left(f_{1}\right)-\left(f_{4}\right)$ and $V$ such that $\left(V_{1}\right)$ and $\left(\mathcal{M}_{1}\right)$ hold. Then, for each sequence $\epsilon_{n} \rightarrow 0$, there exists a subsequence still denoted by $\left(\epsilon_{n}\right)$ such that (1.3) (with $\epsilon=\epsilon_{n}$ ) has a nodal bound state $u_{n}$ such that $u\left(x^{\prime}, x^{\prime \prime}\right)=u\left(x^{\prime},\left|x^{\prime \prime}\right|\right)$ and, if $\epsilon_{n} P_{n}^{1}$ and $\epsilon_{n} P_{n}^{2}$ are respectively a minimum and a maximum point of $u_{n}$, then $\epsilon_{n} P_{n}^{i} \in \Omega, i=1,2$, for $n$ sufficiently large,

$$
\epsilon_{n} P_{n}^{i} \rightarrow x_{0}, \quad \text { as } n \rightarrow \infty,
$$

where $\mathcal{M}\left(x_{0}\right)=\mathcal{M}_{0}$ and

$$
\left|u_{n}(x)\right| \leq C\left(e^{-\frac{\beta}{\epsilon_{n}} d_{k}\left(x, \epsilon_{n} P_{n}^{1}\right)}+e^{-\frac{\beta}{\epsilon_{n}} d_{k}\left(x, \epsilon_{n} P_{n}^{2}\right)}\right), \quad x \in \mathbb{R}^{N},
$$

where $C, \beta>0$ and $d_{k}$ is the distance defined in (1.5). 
The arguments in proving the existence of solutions were strongly influenced by the works of Alves and Souto [17], in which they prove the existence of nodal solutions to a Schrödinger-Poisson system. In the concentration, we follow closely the arguments in [14] and $[12,13]$.

After our work has been finished, we found a very recent paper [18] in which the author uses a similar argumentation in order to prove the existence of a sequence of nodal multi-peak solutions which concentrate around the minimum points of a modified potential, associated to a vanishing potential. The existence arguments in both works rely on a minimization of the penalized energy functional on the nodal Nehari set, and the concentration arguments follow the same general lines. Nevertheless, it is worth pointing out that in our work, since we get sphere-concentrating solutions, several technical difficulties arise. Moreover, in our work proving that the solution of the modified problem is in fact a solution of the original one involves different comparison functions since our penalization is slightly different.

In Section 2 we present the penalization scheme and the variational framework. In Section 3 we prove the existence of nodal solutions of the modified problem. In Section 4 we exhibit the concentration arguments in order to prove that the solutions of the modified problem concentrate around a $k$-dimensional sphere; and in the last section we complete the proof of Theorem 1.1 by showing that the solutions of the modified problem satisfy the original one.

\section{The penalized nonlinearity and the variational framework}

The penalization we are going to apply is a variation of the classical method of Del Pino and Felmer in [4], developed by Sato in [16], in order to allow its use in finding nodal solutions. Fixing $2<\tau<\theta$, let $r_{\epsilon}>0$ such that

$$
\frac{f\left(r_{\epsilon}\right)}{r_{\epsilon}}=\epsilon^{\tau} \quad \text { and } \quad \frac{f\left(-r_{\epsilon}\right)}{-r_{\epsilon}}=\epsilon^{\tau} .
$$

Since $r_{\epsilon} \rightarrow 0$ as $\epsilon \rightarrow 0,\left(f_{1}\right)$ implies that

$$
\epsilon^{\tau}=\frac{f\left(\left|r_{\epsilon}\right|\right)}{\left|r_{\epsilon}\right|} \leq\left|r_{\epsilon}\right|^{\nu-1}
$$

Thus $\epsilon^{\frac{\tau}{v-1}} \leq\left|r_{\epsilon}\right|$, and we can choose an odd function $\tilde{f}_{\epsilon} \in C^{1}(\mathbb{R})$ satisfying

$$
\begin{aligned}
& \tilde{f}_{\epsilon}(s)= \begin{cases}f(s) & \text { if }|s| \leq \frac{1}{2} \epsilon^{\frac{\tau}{v-1}}, \\
\epsilon^{\tau} s & \text { if }|s| \geq \epsilon^{\frac{\tau}{v-1}},\end{cases} \\
& \left|\tilde{f}_{\epsilon}(s)\right| \leq \epsilon^{\tau}|s| \quad \text { for all } s \in \mathbb{R}, \\
& 0 \leq \tilde{f}_{\epsilon}^{\prime}(s) \leq 2 \epsilon^{\tau} \quad \text { for all } s \in \mathbb{R}
\end{aligned}
$$

and

$$
s \mapsto \tilde{f}_{\epsilon}(s) / s \text { is increasing for } s>0 \text { and decreasing for } s<0 .
$$


Let us define $g_{\epsilon}(x, s):=\chi_{\Omega}(x) f(s)+\left(1-\chi_{\Omega}(x)\right) \tilde{f}_{\epsilon}(s)$, where $\chi_{\Omega}$ is the characteristic function of $\Omega$. Note that by $\left(f_{1}\right)-\left(f_{4}\right), g$ is a Charathéodory function such that $g_{\epsilon}\left(x^{\prime}, x^{\prime \prime}, s\right)=$ $g_{\epsilon}\left(x^{\prime},\left|x^{\prime \prime}\right|, s\right)$ satisfying

$\left(g_{1}\right) g_{\epsilon}(x, s)=o\left(|s|^{\nu}\right)$, as $s \rightarrow 0$, uniformly in compact sets of $\mathbb{R}^{N}$.

$\left(g_{2}\right)$ There exist $c_{1}, c_{2}>0$ such that $\left|g_{\epsilon}(x, s)\right| \leq c_{1}|s|+c_{2}|s|^{p}$, where $1<p<\frac{N+2}{N-2}$;

$\left(g_{3}\right)$ There exists $\theta>2$ such that:

(i) $0<\theta G_{\epsilon}(x, s) \leq g_{\epsilon}(x, s) s$ for $x \in \Omega$ and $s \neq 0$,

(ii) $0<2 G_{\epsilon}(x, s) \leq g_{\epsilon}(x, s) s$ for $x \in \mathbb{R}^{N} \backslash \Omega$ and $s \neq 0$, where $G_{\epsilon}(x, s)=\int_{0}^{s} g_{\epsilon}(x, t) d t$.

$\left(g_{4}\right) s \mapsto \frac{g_{\epsilon}(x, s)}{s}$ is a nondecreasing function for $s>0$ and nonincreasing for $s<0$, for all $x \in \mathbb{R}^{N}$.

In a first moment, the concern will be with the penalized problem

$$
-\epsilon^{2} \Delta u+V(x) u=g_{\epsilon}(x, u) \quad \text { in } \mathbb{R}^{N} .
$$

Taking $v_{\epsilon}(x)=u_{\epsilon}(\epsilon x)$, we relate each solution $u_{\epsilon}$ of $(2.4)$ with a solution $v_{\epsilon}$ of

$$
-\Delta v+V(\epsilon x) v=g_{\epsilon}(\epsilon x, u) \quad \text { in } \mathbb{R}^{N}
$$

In order to obtain solutions of (2.5) with a partial symmetry, let us consider the following subspace of $H^{1}\left(\mathbb{R}^{N}\right)$ :

$$
\tilde{H}:=\left\{v \in H^{1}\left(\mathbb{R}^{N}\right) ; \int\left(|\nabla v|^{2}+V(\epsilon x) v^{2}\right)<+\infty \text { and } v\left(x^{\prime}, x^{\prime \prime}\right)=v\left(x^{\prime},\left|x^{\prime \prime}\right|\right)\right\},
$$

which is a Hilbert space when endowed with the inner product

$$
\langle u, v\rangle_{\epsilon}=\left(\int(\nabla u \nabla v+V(\epsilon x) u v)\right),
$$

which gives rise to the following norm:

$$
\|v\|_{\epsilon}=\left(\int\left(|\nabla v|^{2}+V(\epsilon x) v^{2}\right)\right)^{\frac{1}{2}} .
$$

Since the approach is variational, let us consider the energy functional $I_{\epsilon}: \tilde{H} \rightarrow \mathbb{R}$, whose Euler-Lagrange equation is (2.5), given by

$$
I_{\epsilon}(v)=\frac{1}{2} \int\left(|\nabla v|^{2}+V(\epsilon x) v^{2}\right)-\int G_{\epsilon}(\epsilon x, v) .
$$

By standard arguments, one can prove that $I_{\epsilon} \in C^{2}(\tilde{H}, \mathbb{R})$.

Remark 2.1 In this section and throughout the rest of the paper, we omit the $d x$ in all the integrals and, when the domain over which the integral is calculated is $\mathbb{R}^{N}$, we write $\int$ rather than $\int_{\mathbb{R}^{N}}$. 


\section{Existence results}

Let us consider the Nehari manifold associated to (2.5), which is well defined by $\left(g_{4}\right)$ and given by

$$
\mathcal{N}_{\epsilon}=\left\{v \in \tilde{H} \backslash\{0\} ; I_{\epsilon}^{\prime}(v) v=0\right\}
$$

Since we are looking for nodal solutions, let us consider the so-called nodal Nehari set

$$
\mathcal{N}_{\epsilon}^{ \pm}=\left\{v \in \tilde{H} ; v^{ \pm} \neq 0 \text { and } I_{\epsilon}^{\prime}(v) v^{ \pm}=0\right\}
$$

Although $\mathcal{N}_{\epsilon}^{ \pm}$is not a manifold since $u \mapsto u^{+}$in $H^{1}\left(\mathbb{R}^{N}\right)$ lacks differentiability, it is a set which contains all nodal solutions of (2.5).

In the next result we try to infer information of $I_{\epsilon}$ with respect to $\mathcal{N}_{\epsilon}^{ \pm}$in the same way that one is used to do with $\mathcal{N}_{\epsilon}$.

Lemma 3.1 Let $v \in \tilde{H}$ such that $v^{ \pm} \neq 0$. Then there exist $t, s>0$ such that $t v^{+}+s v^{-} \in \mathcal{N}_{\epsilon}^{ \pm}$.

Proof First of all, let us prove that, for all $v \in \tilde{H} \backslash\{0\}$, there exists $t>0$ such that $t v \in \mathcal{N}_{\epsilon}$. Indeed, if $\Gamma=\left(\operatorname{supp} v \cap \Omega_{\epsilon}\right) \cup\left\{x \in \mathbb{R}^{N} ;|v(x)| \leq \frac{1}{2} \epsilon^{\frac{\tau}{v-1}}\right\}$, where $\Omega_{\epsilon}=\epsilon^{-1} \Omega$ and $|\Gamma|$ denotes the $N$-dimensional Lebesgue measure of $\Gamma$, note that $|\Gamma|>0\left(\right.$ since $\left.v \in H^{1}\left(\mathbb{R}^{N}\right)\right)$ and then

$$
\begin{aligned}
I_{\epsilon}(t v) & =\frac{t^{2}}{2}\|v\|_{\epsilon}^{2}-\int_{\Gamma} F(t v)-\int_{\mathbb{R}^{n} \backslash \Gamma} G(\epsilon x, v) \\
& \leq \frac{t^{2}}{2}\|v\|_{\epsilon}^{2}-t^{\theta} \int_{\Gamma}|v|^{\theta}-\frac{t^{\tau}}{2} \int_{\mathbb{R}^{n} \backslash \Gamma}|v| \\
& \rightarrow-\infty,
\end{aligned}
$$

as $t \rightarrow \infty$. Then, if $v \in \tilde{H}$ is such that $v^{ \pm} \neq 0$, there exist $t, s>0$ such that

$$
I_{\epsilon}^{\prime}\left(t v^{+}\right) t v^{+}=0 \quad \text { and } \quad I_{\epsilon}^{\prime}\left(s v^{-}\right) s v^{-}=0 .
$$

Then it is clear that

$$
I_{\epsilon}^{\prime}\left(t v^{+}+s v^{-}\right)\left(t v^{+}+s v^{-}\right)=I_{\epsilon}^{\prime}\left(t v^{+}\right) t v^{+}+I_{\epsilon}^{\prime}\left(s v^{-}\right) s v^{-}=0 .
$$

For a fixed $v \in \tilde{H}$, let us consider $\psi_{v}:[0,+\infty) \times[0,+\infty) \rightarrow \mathbb{R}$ given by

$$
\psi_{v}(t, s)=I_{\epsilon}\left(t v^{+}+s v^{-}\right)
$$

and note that by the smoothness of $g, \psi_{\nu} \in C^{2}\left(\mathbb{R}^{2}, \mathbb{R}\right)$.

Lemma 3.2 Let $v \in \mathcal{N}_{\epsilon}^{ \pm}$, then $(t, s)=(1,1)$ is a strict global maximum point of $\psi_{v}$.

Proof First of all let us note that if $v \in \mathcal{N}_{\epsilon}^{ \pm}$, by $\left(g_{3}\right)$,

$$
\lim _{|(t, s)| \rightarrow \infty} \psi_{\nu}(t, s)=-\infty
$$


Then there exists $R>0$ such that

$$
\psi_{\nu}(t, s)<0 \quad \text { if }|(t, s)| \geq R
$$

Since

$$
\nabla \psi_{v}(t, s)=\left(I_{\epsilon}^{\prime}\left(t v^{+}\right) v^{+}, I_{\epsilon}^{\prime}\left(s v^{-}\right) v^{-}\right)
$$

standard calculations about the behavior of $t \mapsto I_{\epsilon}\left(t v^{+}\right)$and $s \mapsto I_{\epsilon}\left(s v^{-}\right)$and the fact that $v \in \mathcal{N}_{\epsilon}^{ \pm}$imply that $\psi_{v}$ has just one critical point given by $(t, s)=(1,1)$.

As we prove in Lemma 3.5 (which is totally independent of this one), $\psi_{v}(1,1)=I_{\epsilon}(v) \geq$ $\rho>0$. By (3.1), in order to get the result, it is enough to prove that $(1,1)$ is a local maximum point of $\psi_{v}$. Note that

$$
D^{2} \psi_{\nu}(t, s)=\left(\begin{array}{cc}
I_{\epsilon}^{\prime \prime}\left(t v^{+}\right)\left(v^{+}, v^{+}\right) & 0 \\
0 & I_{\epsilon}^{\prime \prime}\left(s v^{-}\right)\left(v^{-}, v^{-}\right)
\end{array}\right)
$$

and then

$$
\begin{aligned}
\operatorname{det}\left(D^{2} \psi_{v}(1,1)\right)= & I_{\epsilon}^{\prime \prime}\left(v^{+}\right)\left(v^{+}, v^{+}\right) \cdot I_{\epsilon}^{\prime \prime}\left(v^{-}\right)\left(v^{-}, v^{-}\right) \\
= & \left(\int\left(g_{\epsilon}\left(\epsilon x, v^{+}\right) v^{+}-g_{\epsilon}^{\prime}\left(\epsilon \mathcal{X}, v^{+}\right) v^{+2}\right)\right) \\
& \cdot\left(\int\left(g_{\epsilon}\left(\epsilon x, v^{-}\right) v^{-}-g_{\epsilon}^{\prime}\left(\epsilon \mathcal{X}, v^{-}\right) v^{-2}\right)\right) .
\end{aligned}
$$

By definition of $g_{\epsilon}$ and $\left(g_{4}\right)$, the last integral is greater or equal to

$$
\begin{aligned}
& \left(\int_{\left(\operatorname{supp}\left(v^{+}\right) \cap \Omega_{\epsilon}\right) \cup\left\{\left|v^{+}\right| \leq \frac{1}{2} \in \frac{\tau}{\left.v^{-1}\right\}}\right.}\left(f\left(v^{+}\right) v^{+}-f^{\prime}\left(v^{+}\right) v^{+2}\right)\right) \\
& \cdot\left(\int_{\operatorname{supp}\left(v^{-}\right) \cap\left(\Omega_{\epsilon} \cup\left\{\left|v^{-}\right|<\frac{1}{2} \epsilon \frac{\tau}{\nu-1}\right\}\right)}\left(f\left(v^{-}\right) v^{-}-f^{\prime}\left(x, v^{-}\right) v^{-2}\right)\right)
\end{aligned}
$$

$>0$.

In the last inequality we have used that by $\left(f_{4}\right)$,

$$
f(s) s-f^{\prime}(s) s^{2}<0 \quad \text { for all } s \neq 0
$$

and as $v^{ \pm} \in \tilde{H},\left|\operatorname{supp}\left(v^{+}\right) \cap\left(\Omega_{\epsilon} \cup\left\{\left|v^{+}\right|<a\right\}\right)\right|>0$ and $\left|\operatorname{supp}\left(v^{-}\right) \cap\left(\Omega_{\epsilon} \cup\left\{\left|v^{-}\right|<a\right\}\right)\right|>0$, where $\Omega_{\epsilon}:=\epsilon^{-1} \Omega$.

Since $D^{2} \psi_{v}(1,1)$ is a positive definite form, we just have to verify that $\frac{\partial^{2} \psi_{v}}{\partial t^{2}}=$ $I_{\epsilon}^{\prime \prime}\left(t v^{+}\right) v^{+2}<0$. But this follows since 1 is a maximum point of $t \mapsto I_{\epsilon}\left(t v^{+}\right)$.

Still, as a consequence of the arguments employed in the construction of the Nehari manifold as in [2], we have the following result. 
Lemma 3.3 Let $v \in \tilde{H}$ such that $v^{ \pm} \neq 0$ and

$$
I_{\epsilon}^{\prime}(v) v^{ \pm} \leq 0,
$$

then there exist $t, s \in(0,1]$ such that

$$
t v^{+}+s v^{-} \in \mathcal{N}_{\epsilon}^{ \pm} .
$$

Proof In fact, let $t \in \mathbb{R}$ such that $I_{\epsilon}^{\prime}\left(t v^{+}\right) t v^{+}=0$. Suppose by contradiction that $t>1$, then

$$
\begin{aligned}
\left\|v^{+}\right\|^{2}= & \int \frac{g_{\epsilon}\left(\epsilon x, t v^{+}\right) v^{+}}{t}=\int_{v^{+}>0} \frac{g_{\epsilon}\left(\epsilon x, t v^{+}\right) v^{+2}}{t v^{+}} \\
= & \int_{\operatorname{supp}\left(v^{+}\right) \cap\left(\Omega_{\epsilon} \cup\left\{\left|t v^{+}\right|<\frac{1}{2} \epsilon \frac{\tau}{\nu-1}\right\}\right)} \frac{f\left(t v^{+}\right) t v^{+2}}{v^{+}} \\
& +\int_{\operatorname{supp}\left(v^{+}\right) \cap\left(\Omega_{\epsilon} \cup\left\{\left|t v^{+}\right| \geq \frac{1}{2} \epsilon \frac{\tau}{\nu-1}\right\}\right)} \frac{g_{\epsilon}\left(\epsilon x, t v^{+}\right) v^{+2}}{t v^{+}} \\
> & \int_{\operatorname{supp}\left(v^{+}\right) \cap\left(\Omega_{\epsilon} \cup\left\{\left|t v^{+}\right|<\frac{1}{2} \epsilon \frac{\tau}{v-1}\right\}\right)} f\left(v^{+}\right) v^{+} \\
& +\int_{\operatorname{supp}\left(v^{+}\right) \cap\left(\Omega_{\epsilon} \cup\left\{\left|v^{+}\right| \geq \frac{1}{2} \frac{\tau}{\nu-1}\right\}\right)} \frac{g_{\epsilon}\left(\epsilon x, v^{+}\right) \nu^{+2}}{v^{+}} \\
= & \int g_{\epsilon}\left(\epsilon x, v^{+}\right) v^{+},
\end{aligned}
$$

which implies that $I_{\epsilon}^{\prime}(v) v^{+}>0$, contradicting the hypothesis. The same argument applies to $v^{-}$and $s$.

Let us define

$$
d_{\epsilon}:=\inf _{\mathcal{N}_{\epsilon}^{ \pm}} I_{\epsilon},
$$

and note that if there exists a solution of (2.5) in the energy level $d_{\epsilon}$, then it is the solution with least energy among all nodal ones.

Now we are going to state and prove the main result of this section.

Theorem 3.4 For sufficiently small $\epsilon>0$, there exists a nodal solution of $(2.5), v_{\epsilon} \in \tilde{H}$ such that $I_{\epsilon}\left(v_{\epsilon}\right)=d_{\epsilon}$.

Before proceeding with the proof of Theorem 3.4, let us state some technical result about $\mathcal{N}_{\epsilon}^{ \pm}$.

\section{Lemma 3.5 It holds that}

(i) There exists $\rho>0$ such that $\|v\|_{\epsilon} \geq \rho$ for all $v \in \mathcal{N}_{\epsilon}$.

(ii) There exists a constant $C>0$ such that, for all $v \in \mathcal{N}_{\epsilon}, I_{\epsilon}(v) \geq C\|v\|_{\epsilon}^{2}$. 
Proof The proof of (i) follows by standard arguments. Let us prove just (ii), which in fact also follows by very well known arguments. Note that if $v \in \mathcal{N}_{\epsilon}$, by $\left(g_{3}\right)$,

$$
\begin{aligned}
I_{\epsilon}(v) & =I_{\epsilon}(v)-\frac{1}{\theta} I^{\prime} \epsilon(v) v=\left(\frac{1}{2}-\frac{1}{\theta}\right)\|v\|_{\epsilon}^{2}+\frac{1}{\theta} \int_{\mathbb{R}^{N}}(g(x, v) v-\theta G(x, v)) d x \\
& \geq\left(\frac{1}{2}-\frac{1}{\theta}\right)\|v\|_{\epsilon}^{2}+\frac{1}{\theta} \int_{\Omega_{\epsilon}^{c}}\left(g\left(\epsilon x, v_{n}\right) v-\theta G(\epsilon x, v)\right) d x \\
& \geq\left(\frac{1}{2}-\frac{1}{\theta}\right)\|v\|_{\epsilon}^{2}+\frac{(2-\theta)}{\theta} \int_{\Omega_{\epsilon}^{c}} G\left(x, u_{n}\right) d x \\
& \geq\left(\frac{1}{2}-\frac{1}{\theta}\right)\|v\|_{\epsilon}^{2}+\frac{(2-\theta)}{2 \theta} \int_{\Omega_{\epsilon}^{c}} \epsilon^{\tau} v^{2} d x \\
& =\left(\frac{\theta-2}{2 \theta}\right) \int_{\mathbb{R}^{N}}\left(|\nabla v|^{2}+\left(V(\epsilon x)-\epsilon^{\tau}\right) v^{2}\right) d x \\
& =\left(\frac{\theta-2}{2 \theta}\right) \int_{\mathbb{R}^{N}}\left(|\nabla v|^{2}+\left(V_{0}-\epsilon^{\tau}\right) v^{2}\right) d x \\
& \geq C\|v\|_{\epsilon}^{2},
\end{aligned}
$$

where $C>0$ for $\epsilon>0$ sufficiently small.

Proof of Theorem 3.4 The proof will be carried out in two steps. In the first one we prove that $d_{\epsilon}$ is attained by a function $u_{\epsilon} \in \tilde{H}$.

Let $\left(w_{n}\right)$ be a minimizing sequence for $I_{\epsilon}$ in $\mathcal{N}_{\epsilon}^{ \pm}$, i.e., a sequence $\left(w_{n}\right) \subset \mathcal{N}_{\epsilon}^{ \pm}$such that

$$
\lim _{n \rightarrow \infty} I_{\epsilon}\left(w_{n}\right)=d_{\epsilon}
$$

Note that, by (3.2) and Lemma 3.5, $\left(w_{n}\right)$ is a bounded sequence in $\tilde{H}$. Then there exists $w_{\epsilon}$ such that $w_{n} \rightarrow w_{\epsilon}$ in $\tilde{H}$ up to a subsequence. In the same way as in Lemma 2.3 in [19], it is possible to show that $v \mapsto v^{ \pm}$is a continuous function of $\tilde{H}$ into itself, from which it follows that $w_{n}^{ \pm} \rightarrow w_{\epsilon}^{ \pm}$in $\tilde{H}$. As a consequence, up to a subsequence

$$
w_{n}^{ \pm} \rightarrow w_{\epsilon}^{ \pm} \quad \text { a.e. in } \mathbb{R}^{N}
$$

and

$$
w_{n}^{ \pm} \rightarrow w_{\epsilon}^{ \pm} \quad \text { in } L^{r}\left(\Omega_{\epsilon}\right) \text { for } 1 \leq r<\frac{2 N}{N-2},
$$

where $w_{\epsilon}^{ \pm} \neq 0$ by the same arguments as in Lemma 2.8 in [16].

Since $w_{\epsilon}^{ \pm} \neq 0$, let $t_{\epsilon}, s_{\epsilon}>0$ be such that $t_{\epsilon} w_{\epsilon}^{+}+s_{\epsilon} w_{\epsilon}^{-} \in \mathcal{N}_{\epsilon}^{ \pm}$. By weak lower-semicontinuity of $\|\cdot\|$ and by Sobolev embeddings, it follows that

$$
\left\|t_{\epsilon} w_{\epsilon}^{+}+s_{\epsilon} w_{\epsilon}^{-}\right\|_{\epsilon} \leq \liminf _{n \rightarrow \infty}\left\|t_{\epsilon} w_{n}^{+}+s_{\epsilon} w_{n}^{-}\right\|_{\epsilon}
$$

and

$$
\int_{\Omega_{\epsilon}} F\left(t_{\epsilon} w_{\epsilon}^{+}+s_{\epsilon} w_{\epsilon}^{-}\right)=\liminf _{n \rightarrow \infty} \int_{\Omega_{\epsilon}} F\left(t_{\epsilon} w_{n}^{+}+s_{\epsilon} w_{n}^{-}\right) .
$$


Now the real essence of the modification of penalization really comes up. Note that for $\epsilon>0$ sufficiently small,

$$
I_{\epsilon, \mathbb{R}^{N} \backslash \Omega_{\epsilon}}(v):=\frac{1}{2} \int_{\mathbb{R}^{N} \backslash \Omega_{\epsilon}}\left(|\nabla v|^{2}+V(\epsilon x) v^{2}\right)-\int_{\mathbb{R}^{N} \backslash \Omega_{\epsilon}} \tilde{F}_{\epsilon}(v)
$$

is a strictly convex functional in $\tilde{H}\left(\mathbb{R}^{n} \backslash \Omega_{\epsilon}\right)=\left\{v \in H^{1}\left(\mathbb{R}^{N} \backslash \Omega_{\epsilon}\right), \int_{\mathbb{R}^{N} \backslash \Omega_{\epsilon}}\left(|\nabla v|^{2}+V(\epsilon x) v^{2}\right)<\right.$ $\infty$ \}. In fact, for $v, h \in \tilde{H}\left(\mathbb{R}^{N} \backslash \Omega_{\epsilon}\right), h \neq 0$, by (2.2) and Sobolev embeddings,

$$
\begin{aligned}
I_{\epsilon, \mathbb{R}^{N} \backslash \Omega_{\epsilon}}^{\prime \prime}(v)(h, h) & =\|h\|_{\epsilon, \mathbb{R}^{N} \backslash \Omega_{\epsilon}}^{2}-\int_{\mathbb{R}^{n} \backslash \Omega_{\epsilon}} \tilde{f}_{\epsilon}^{\prime}(v) h^{2} \\
& \geq\|h\|_{\epsilon, \mathbb{R}^{N} \backslash \Omega_{\epsilon}}^{2}\left(1-\epsilon^{\tau}\right)>0
\end{aligned}
$$

for $\epsilon>0$ sufficiently small. Then by convex analysis it follows that $I_{\epsilon, \mathbb{R}^{N} \backslash \Omega_{\epsilon}}$ is weakly lower semicontinuous. Then (3.5), (3.6) and this fact imply that

$$
\begin{aligned}
I_{\epsilon}\left(t_{\epsilon} w_{\epsilon}^{+}+s_{\epsilon} w_{\epsilon}^{-}\right) & \leq \liminf _{n \rightarrow \infty} I_{\epsilon}\left(t_{\epsilon} w_{\epsilon}^{+}+s_{\epsilon} w_{\epsilon}^{-}\right) \\
& \leq \liminf _{n \rightarrow \infty}\left(I_{\epsilon}\left(t_{\epsilon} w_{\epsilon}^{+}\right)+I_{\epsilon}\left(s_{\epsilon} w_{\epsilon}^{-}\right)\right) \\
& \leq \liminf _{n \rightarrow \infty}\left(I_{\epsilon}\left(w_{\epsilon}^{+}\right)+I_{\epsilon}\left(w_{\epsilon}^{-}\right)\right) \\
& =b_{\epsilon} .
\end{aligned}
$$

Hence $I_{\epsilon}\left(t_{\epsilon} w_{\epsilon}^{+}+s_{\epsilon} w_{\epsilon}^{-}\right)=b_{\epsilon}$.

The second step is proving that $v_{\epsilon} \in \tilde{H}$, which minimizes $I_{\epsilon}$ on $\mathcal{N}_{\epsilon}^{ \pm}$is a critical point of $I_{\epsilon}$ in $\tilde{H}$. This can be done by employing the same arguments as those of Section 3 in [20]. For the sake of completeness, we include all the details of this proof.

Supposing by contradiction that $I_{\epsilon}^{\prime}\left(v_{\epsilon}\right) \neq 0$, there exist $\delta, \lambda>0$ such that

$$
\left\|I_{\epsilon}^{\prime}(v)\right\|_{*} \geq \lambda \quad \text { for all } v \in B_{\delta}\left(v_{\epsilon}\right) \subset \tilde{H} .
$$

Let us consider the function $(t, s) \mapsto t v_{\epsilon}^{+}+s v_{\epsilon}^{-}$defined on $D=\left(\frac{1}{2}, \frac{3}{2}\right)^{2}$, and note that by Lemma 3.2

$$
\delta_{\epsilon}:=\max _{(t, s) \in \partial D} I_{\epsilon}\left(t v_{\epsilon}^{+}+s v_{\epsilon}^{-}\right)<I_{\epsilon}\left(v_{\epsilon}\right)=d_{\epsilon} .
$$

Taking $\rho=\min \left\{\frac{d_{\epsilon}-\delta_{\epsilon}}{2}, \frac{\lambda \delta}{24}\right\}$ and $S=B_{\delta / 3}\left(v_{\epsilon}\right)$, Lemma 2.3 in [21] yields a deformation $\eta$ such that

(i) $\eta(1, v)=v$ for all $v \notin I_{\epsilon}^{-1}\left(\left[d_{\epsilon}-2 \rho, d_{\epsilon}+2 \rho\right]\right)$,

(ii) $\eta\left(1, I_{\epsilon}^{d_{\epsilon}+\rho} \cap S\right) \subset I_{\epsilon}^{d_{\epsilon}-\rho}$,

(iii) $I_{\epsilon}(\eta(1, v)) \leq I_{\epsilon}(v)$ for all $u \in \tilde{H}$.

Claim.

$$
\max _{(t, s) \in \bar{D}} I_{\epsilon}\left(\eta\left(1, t v_{\epsilon}^{+}+s v_{\epsilon}^{-}\right)\right)<d_{\epsilon} .
$$

In fact, if $(t, s) \neq(1,1)$, then by Lemma 3.2,

$$
I_{\epsilon}\left(\eta\left(1, t v_{\epsilon}^{+}+s v_{\epsilon}^{-}\right)\right) \leq I_{\epsilon}\left(t v_{\epsilon}^{+}+s v_{\epsilon}^{-}\right)<d_{\epsilon} .
$$


On the other hand, by (ii),

$$
I_{\epsilon}\left(\eta\left(1, v_{\epsilon}\right)\right)<d_{\epsilon}-\rho
$$

which proves the claim.

Now, let us prove that there exists $(t, s) \in D$ such that $\eta\left(1, t v_{\epsilon}^{+}+s v_{\epsilon}^{-}\right) \in \mathcal{N}_{\epsilon}^{ \pm}$, which together with the claim contradicts the definition of $d_{\epsilon}$.

Define $h(t, s)=\eta\left(1, t v_{\epsilon}^{+}+s v_{\epsilon}^{-}\right)$and $\Psi_{0}, \Psi_{1}: \mathbb{R}^{2} \rightarrow \mathbb{R}^{2}$ by

$$
\Psi_{0}(t, s)=\left(I_{\epsilon}^{\prime}\left(t v_{\epsilon}^{+}\right) v_{\epsilon}^{+}, I_{\epsilon}^{\prime}\left(s v_{\epsilon}^{-}\right) v_{\epsilon}^{-}\right)
$$

and

$$
\Psi_{1}(t, s)=\left(\frac{1}{t} I_{\epsilon}^{\prime}\left(h(t, s)^{+}\right) h(t, s)^{+}, \frac{1}{s} I_{\epsilon}^{\prime}\left(h(t, s)^{-}\right) h(t, s)^{-}\right) .
$$

By results of Brouwer degree theory, $\operatorname{deg}\left(\Psi_{0}, D,(0,0)\right)=1$. On the other hand, note that by (3.8), $(t, s) \mapsto t v_{\epsilon}^{+}+s v_{\epsilon}^{-}$coincides with $h$ on $\partial D$. Hence $\Psi_{0}=\Psi_{1}$ on $\partial D$, and then $\operatorname{deg}\left(\Psi_{1}, D,(0,0)\right)=\operatorname{deg}\left(\Psi_{0}, D,(0,0)\right)=1$. Therefore there exists $(t, s) \in D$ such that $\Psi_{1}(t, s)=$ $(0,0)$ and, consequently,

$$
\eta\left(1, t v_{\epsilon}^{+}+s v_{\epsilon}^{-}\right)=h(t, s) \in \mathcal{N}_{\epsilon}^{ \pm}
$$

Finally, this contradiction proves the theorem.

\section{Concentration results}

Let us introduce a sequence $\epsilon_{n} \rightarrow 0$ as $n \rightarrow \infty$ and, for each $n \in \mathbb{N}$, let us denote by $v_{n}$ the solution $v_{\epsilon_{n}}$ given by Theorem 3.4 and consider $d_{n}:=d_{\epsilon_{n}},\|\cdot\|_{n}:=\|\cdot\|_{\epsilon_{n}}$ and $I_{n}:=I_{\epsilon_{n}}$.

The following result provides an upper estimate for the sequence of values $d_{n}$. Its proof is inspired by the arguments of Alves and Soares in [14].

\section{Lemma 4.1}

$$
\limsup _{n \rightarrow \infty} \epsilon_{n}^{k} d_{n} \leq 2 \omega_{k} \inf _{\Omega} \mathcal{M}
$$

and

$$
\epsilon_{n}^{k}\left\|v_{n}\right\|_{n}^{2} \leq C
$$

where $\omega_{k}$ denotes the volume of the unitary $k$-dimensional ball on $\mathbb{R}^{N}$.

Proof Let $z_{0}=\left(x_{0}, y_{0}\right) \in \Omega$ be such that $\mathcal{M}\left(z_{0}\right)=\inf _{x \in \Omega} \mathcal{M}(x)$. Since $\Omega$ is an open set, there exists $R>0$ such that $B_{2 R}\left(z_{0}\right) \subset \Omega$. Let us choose points $z_{1}, z_{2} \in \partial B_{R}\left(z_{0}\right)$ such that, if $z_{i}=\left(Q_{i}^{\prime}, Q_{i}^{\prime \prime}\right)$, then $\left|Q_{1}^{\prime \prime}-Q_{2}^{\prime \prime}\right|=2 R$. Note that $B_{R}\left(z_{i}\right) \subset \Omega$ for $i=1,2$. In the rest of this proof, $i \in\{1,2\}$. Let us choose smooth cut-off functions $\psi_{i}: \mathbb{R}^{N-k} \rightarrow \mathbb{R}$ such that $\psi_{i}=1$ in $B_{\mathbb{R}^{N-k}}\left(\left(Q_{i}^{\prime},\left|Q_{i}^{\prime \prime}\right|\right), R / 4\right)$ and $\psi_{i}=0$ in $\mathbb{R}^{N-k} \backslash B_{\mathbb{R}^{N-k}}\left(\left(Q_{i}^{\prime},\left|Q_{i}^{\prime \prime}\right|\right), R / 2\right)$. 
Let $w_{i} \in H^{1}\left(\mathbb{R}^{N-k}\right)$ be a ground-state solution of

$$
-\Delta u+V\left(z_{i}\right) u=f(u) \quad \text { in } \mathbb{R}^{N-k}
$$

and $w_{n, z_{i}}: \mathbb{R}^{N} \rightarrow \mathbb{R}$ be given by

$$
w_{n, z_{i}}\left(x^{\prime}, x^{\prime \prime}\right)=\psi_{i}\left(\epsilon_{n} x^{\prime}, \epsilon_{n}\left|x^{\prime \prime}\right|\right) w_{i}\left(x^{\prime}-\frac{Q_{i}^{\prime}}{\epsilon_{n}},\left|x^{\prime \prime}\right|-\frac{\left|Q_{i}^{\prime \prime}\right|}{\epsilon_{n}}\right) .
$$

We associate for each $w_{n, z_{i}}(x)$ its $k$-dimensional counterpart $\tilde{w}_{n, z_{i}}\left(x^{\prime}, r\right)=w_{n, z_{i}}\left(x^{\prime}, x^{\prime \prime}\right)$, where $\left|x^{\prime \prime}\right|=r \in \mathbb{R}$. The existence of $t_{i_{n}}>0$ such that $t_{i_{n}} w_{n, z_{i}} \in \mathcal{N}_{\epsilon_{n}}$ is well known. By the construction we have made so far, it is straightforward to see that

$$
\bar{w}_{n}:=t_{1_{n}} w_{n, z_{1}}-t_{2_{n}} w_{n, z_{2}} \in \mathcal{N}_{\epsilon_{n}}^{ \pm} .
$$

Using the fact that $I_{n}$ is even, we have

$$
d_{n} \leq I_{n}\left(\bar{w}_{n}\right)=I_{n}\left(t_{1_{n}} w_{n, z_{1}}\right)+I_{n}\left(t_{2_{n}} w_{n, z_{2}}\right) .
$$

By a change of variable, let us note that for $i \in\{1,2\}, I_{n}\left(t_{n} w_{n, z_{i}}\right)$ is equal to

$$
\begin{aligned}
& \frac{t_{i_{n}}^{2}}{2} \int_{\mathbb{R}^{N}}\left(\left|\nabla w_{n, z_{i}}\right|^{2}+V\left(\epsilon_{n} x\right) w_{n, z_{i}}^{2}\right) d x-\int_{\mathbb{R}^{N}} G_{n}\left(\epsilon_{n} x, t_{i_{n}} w_{n, z_{i}}\right) d x \\
&=\omega_{k}\left[\frac{t_{i_{n}}^{2}}{2} \int_{\mathbb{R}^{N-k-1}} \int_{0}^{+\infty} r^{k}\left(\left|\nabla \tilde{w}_{n, z_{i}}\left(x^{\prime}, r\right)\right|^{2}+V\left(\epsilon_{n} x^{\prime}, \epsilon_{n} r\right) \tilde{w}_{n, z_{i}}\left(x^{\prime}, r\right)^{2}\right) d r d x^{\prime}\right. \\
&\left.\quad-\int_{\mathbb{R}^{N-k-1}} \int_{0}^{+\infty} r^{k} G_{n}\left(\epsilon_{n} x, \epsilon_{n} r, t_{i_{n}} \tilde{w}_{n, z_{i}}\left(x^{\prime}, r\right)\right) d r d x^{\prime}\right] \\
&=\omega_{k}\left[\frac{t_{i_{n}}^{2}}{2} \int_{\mathbb{R}^{N-k-1}} \int_{\frac{-\mid Q_{i}^{\prime \prime}}{\epsilon_{n}}}^{+\infty}\left(\sigma+\frac{\left|Q_{i}^{\prime \prime}\right|}{\epsilon_{n}}\right)^{k}\left|\nabla\left(\psi_{i}\left(\epsilon_{n} x^{\prime}+Q_{i}^{\prime}, \epsilon_{n} \sigma+\left|Q_{i}^{\prime \prime}\right|\right) w_{i}\left(x^{\prime}, \sigma\right)\right)\right|^{2}\right. \\
& \quad+V\left(\epsilon_{n} x^{\prime}+\epsilon_{n} Q_{i}^{\prime}, \epsilon_{n} \sigma+\left|Q_{i}^{\prime \prime}\right|\right)\left(\psi_{i}\left(\epsilon_{n} x^{\prime}+\epsilon_{n} Q_{i}^{\prime}, \epsilon_{n} \sigma+\left|Q_{i}^{\prime \prime}\right|\right) w_{i}\left(x^{\prime}, \sigma\right)^{2}\right) d \sigma d x^{\prime} \\
& \quad-\int_{\frac{-\left|Q_{i}^{\prime \prime}\right|}{\epsilon_{n}}}^{+\infty}\left(\sigma+\frac{\left|Q_{i}^{\prime \prime}\right|}{\epsilon_{n}}\right)^{k} G_{n}\left(\epsilon_{n} x^{\prime}+\epsilon_{n} Q_{i}^{\prime}, \epsilon_{n} \sigma+\left|Q_{i}^{\prime \prime}\right|,\right. \\
&\left.\left.t_{i_{n}} \psi_{i}\left(\epsilon_{n} x^{\prime}+\epsilon_{n} Q_{i}^{\prime}, \epsilon_{n} \sigma+\left|Q_{i}^{\prime \prime}\right|\right) w_{i}\left(x^{\prime}, \sigma\right)\right) d \sigma d x^{\prime}\right] \\
&= \epsilon_{n}^{-k} \omega_{k}\left|Q_{i}\right|^{k} I_{V\left(z_{i}\right)}\left(t_{i_{n}} w_{i}\right)+o(1) \leq \epsilon_{n}^{-k} \omega_{k}\left|Q_{i}\right|^{k} \mathcal{E}\left(V\left(z_{i}\right)\right)=\epsilon_{n}^{-k} \omega_{k} \mathcal{M}\left(z_{i}\right)+o(1) .
\end{aligned}
$$

By the last inequality and (4.1), we have

$$
\epsilon_{n}^{k} d_{n} \leq \omega_{k}\left(\mathcal{M}\left(z_{1}\right)+\mathcal{M}\left(z_{2}\right)\right)+o(1)
$$

and making $R \rightarrow 0$, the continuity of $V$ and $\mathcal{M}$ implies the result. 
Now, in order to see that

$$
\epsilon_{n}^{k}\left\|v_{n}\right\|_{n}^{2} \leq C
$$

just observe that $v_{n} \in \mathcal{N}_{\epsilon_{n}}$ and use (ii) of Lemma 3.5.

The next lemma implies that solutions found in Theorem 3.4 do not vanish when $n \rightarrow \infty$.

Lemma 4.2 Let $P_{n}^{1}, P_{n}^{2}$ be local maximum and minimum points of $v_{n}$, respectively. Then $P_{n}^{i} \in \Omega_{\epsilon_{n}}:=\epsilon_{n}^{-1} \Omega$,

$$
v_{n}\left(P_{n}^{1}\right) \geq a \quad \text { and } \quad v_{n}\left(P_{n}^{2}\right) \leq-a
$$

where $a>0$ is such that $f(a) / a=V_{0} / 2$.

Proof First of all let us prove that $P_{n}^{i} \in \Omega_{\epsilon_{n}}$. Suppose by contradiction that $P_{n}^{1} \notin \Omega_{\epsilon_{n}}$. Since $P_{n}^{1}$ is a local maximum point, it follows that $\Delta v_{n}\left(P_{n}^{1}\right) \leq 0$. By definition of $g_{n}$, we have

$$
V_{0} v_{n}\left(P_{n}^{1}\right) \leq-\Delta v_{n}\left(P_{n}^{1}\right)+V\left(\epsilon_{n} P_{n}^{1}\right) v_{n}\left(P_{n}^{1}\right)=\tilde{f}_{\epsilon_{n}}\left(v_{n}\left(P_{n}^{1}\right)\right) \leq \epsilon_{n}^{\tau} v_{n}\left(P_{n}^{1}\right),
$$

which is impossible for $\epsilon>0$ sufficiently small. A similar argument applies to $P_{n}^{2}$.

Since $P_{n}^{1} \in \Omega_{\epsilon_{n}}$ and $v_{n}\left(P_{n}^{1}\right)>0$, from the definition of $g_{n}$ we have

$$
0 \geq \Delta v_{n}\left(P_{n}^{1}\right)=\left(V\left(\epsilon_{n} P_{n}^{1}\right)-\frac{f\left(v_{n}\left(P_{n}^{1}\right)\right)}{v_{n}\left(P_{n}^{1}\right)}\right) v_{n}\left(P_{n}^{1}\right) .
$$

Supposing by contradiction that $v_{n}\left(P_{n}^{1}\right)<a$. By the choice of $a>0$ and $\left(f_{5}\right)$ it follows that

$$
V_{0} \leq V\left(\epsilon_{n} P_{n}^{1}\right) \leq \frac{f\left(v_{n}\left(P_{n}^{1}\right)\right)}{v_{n}\left(P_{n}^{1}\right)} \leq \frac{f(a)}{a}=\frac{V_{0}}{2},
$$

which is a contradiction. Analogously we prove that $v_{n}\left(P_{n}^{2}\right) \leq-a$.

By the last result, there exist $P^{1}, P^{2} \in \bar{\Omega}$ such that along a subsequence

$$
\lim _{n \rightarrow \infty} \epsilon_{n} P_{n}^{i}=P^{i}, \quad i \in\{1,2\}
$$

The same argument of [14] with short modifications can be used to prove the following result.

Lemma 4.3 Using the same notation as that in the last result, it follows that

$$
\lim _{n \rightarrow \infty}\left|P_{n}^{1}-P_{n}^{2}\right|=+\infty
$$

Lemma 4.4 Let $y_{n}=\left(y_{n}^{\prime}, y_{n}^{\prime \prime}\right) \subset \mathbb{R}^{N}$ be a sequence such that $\epsilon_{n} y_{n} \rightarrow\left(\bar{y}^{\prime}, \bar{y}^{\prime \prime}\right) \in \bar{\Omega}$ as $n \rightarrow \infty$. Denoting $\tilde{v}_{n}\left(x^{\prime}, r\right):=v_{n}\left(x^{\prime}, x^{\prime \prime}\right)$, where $\left|x^{\prime \prime}\right|=r$, let us define $\tilde{w}_{n}: \mathbb{R}^{N-k-1} \times\left[-\left|y_{n}\right|,+\infty\right) \rightarrow \mathbb{R}$ 
by

$$
\tilde{w}_{n}\left(x^{\prime}, r\right):=\tilde{v}_{n}\left(x^{\prime}, r+\left|y_{n}^{\prime \prime}\right|\right)
$$

Then there exists $\tilde{w} \in H^{1}\left(\mathbb{R}^{N-k}\right)$ such that $\tilde{w}_{n} \rightarrow \tilde{w}$ in $C_{l o c}^{2}\left(\mathbb{R}^{N-k}\right)$ and $\tilde{w}$ satisfies the limit problem

$$
-\Delta \tilde{w}+V\left(\bar{y}^{\prime},\left|\bar{y}^{\prime \prime}\right|\right) \tilde{w}=\tilde{g}_{n}\left(x^{\prime}, r, \tilde{w}\right) \quad \text { in } \mathbb{R}^{N-k},
$$

where $\tilde{g}_{n}\left(x^{\prime}, r, s\right):=\chi\left(x^{\prime}, r\right) f(s)+\left(1-\chi\left(x^{\prime}, r\right)\right) \tilde{f}_{\epsilon_{n}}(s)$ and $\chi\left(x^{\prime}, r\right)=\lim _{n \rightarrow \infty} \chi_{\Omega}\left(\epsilon_{n} x^{\prime}+\epsilon_{n} y_{n}^{\prime}\right.$, $\left.\epsilon_{n} r+\epsilon_{n}\left|y_{n}^{\prime \prime}\right|\right)$.

Proof The proof is analogous to [12][Lemma 4.3], but we sketch it here for the sake of completeness.

Note that $\tilde{w}_{n}$ satisfies the following problem:

$$
\begin{aligned}
& -\Delta \tilde{w}_{n}-\frac{k}{\left(r+\left|y_{n}^{\prime \prime}\right|\right)} \frac{\tilde{w}_{n}}{\partial r}+V\left(\epsilon_{n} x^{\prime}+\epsilon_{n} y_{n}^{\prime}, \epsilon_{n} r+\epsilon_{n}\left|y_{n}^{\prime \prime}\right|\right) \tilde{w}_{n} \\
& =g_{n}\left(\epsilon_{n} x^{\prime}+\epsilon_{n} y_{n}^{\prime}, \epsilon_{n} r+\epsilon_{n}\left|y_{n}^{\prime \prime}\right|, \tilde{w}_{n}\right),
\end{aligned}
$$

in $\mathbb{R}^{N-k-1} \times\left[-\left|y_{n}\right|,+\infty\right)$.

By Lemma 4.1 it follows that

$$
\int_{\mathbb{R}^{N-k-1}} \int_{-\left|y_{n}^{\prime \prime}\right|}^{+\infty}\left(\left|\nabla \tilde{w}_{n}\right|^{2}+V\left(\epsilon_{n} x^{\prime}+\epsilon_{n} y_{n}^{\prime}, \epsilon_{n} r+\epsilon_{n}\left|y_{n}^{\prime \prime}\right|\right) \tilde{w}_{n}^{2}\right) d r d x^{\prime} \leq C
$$

uniformly in $n$ and then, for some $\tilde{w} \in H^{1}\left(\mathbb{R}^{N-k}\right)$,

$$
\tilde{w}_{n} \rightarrow \tilde{w} \quad \text { in } H^{1}\left(\mathbb{R}^{N-k}\right) .
$$

By choosing a sequence $R_{n} \rightarrow \infty$ such that $\epsilon_{n} R_{n} \rightarrow 0$ and considering a smooth cutoff function in $\mathbb{R}^{N-k}, \eta_{R}$ such that $0 \leq \eta_{R} \leq 1, \eta_{R}(z)=1$ if $|z| \leq \frac{R}{2}$ and $\eta_{R}(z)=0$ if $|z|>R$, and $\left\|\nabla \eta_{R}\right\|_{\infty} \leq \frac{C}{R}$, it can be proved using (4.8) that $\bar{w}_{n}(z):=\eta_{R_{n}}(z) \tilde{w}_{n}(z)$ is bounded in $H^{1}\left(\mathbb{R}^{N-k}\right)$, uniformly in $n$.

Since $\bar{w}_{n}$ satisfies $(4.7)$ in $B\left(0, R_{n}\right)$, it follows by classical elliptic estimates that

$$
\left\|\bar{w}_{n}\right\|_{W^{2, q}(B(0, R))} \leq C
$$

for sufficiently large $n \in \mathbb{N}$, where $R>0$ is fixed.

By (4.9) and (4.10) it follows that $\tilde{w}_{n} \rightarrow \tilde{w}$ in $C_{l o c}^{2}\left(\mathbb{R}^{N-k}\right)$ and that $\tilde{w}$ satisfies (4.6).

Since the concentration set is expected to be a sphere in $\mathbb{R}^{N}$, it is natural to introduce the distance between two $k$-dimensional spheres in $\mathbb{R}^{N}$, which gives rise to neighborhoods in which we want to estimate the mass of solutions. Let us recall that for $x=\left(x^{\prime}, x^{\prime \prime}\right), y=$ $\left(y^{\prime}, y^{\prime \prime}\right) \in \mathbb{R}^{N}$,

$$
d_{k}(x, y)=\sqrt{\left(x^{\prime}-y^{\prime}\right)^{2}+\left(\left|x^{\prime \prime}\right|-\left|y^{\prime \prime}\right|\right)^{2}}
$$


denotes the distance between $k$-dimensional spheres centered at the origin, parallel to $\mathcal{H}^{\perp}$ and of radius $\left|x^{\prime \prime}\right|$ and $\left|y^{\prime \prime}\right|$, respectively. According to this distance, the balls are given by

$$
B_{k}(x, r)=\left\{y \in \mathbb{R}^{N} ; d_{k}(x, y)<r\right\} .
$$

From now on, for $\Lambda \subset \mathbb{R}^{N}$, we denote

$$
I_{n, \Lambda}(v)=\frac{1}{2} \int_{\Lambda}\left(|\nabla v|^{2}+V\left(\epsilon_{n} x\right) v^{2}\right) d x-\int_{\Lambda} G_{n}\left(\epsilon_{n} x, v\right) d x
$$

The following is the main step in proving the concentration result.

\section{Proposition 4.5 Suppose that all the assumptions of Theorem $1.1 \mathrm{hold}$. Then}

(i) $\lim _{n \rightarrow \infty} \epsilon_{n}^{k} d_{n}=2 \omega_{k} \inf _{\Omega} \mathcal{M}$;

(ii) $\lim _{n \rightarrow \infty} \mathcal{M}\left(\epsilon_{n} P_{n}^{i}\right)=\inf _{\Omega} \mathcal{M}, i \in\{1,2\}$.

Proof Let us get started with (i). By Lemma 4.1 it follows that

$$
\limsup _{n \rightarrow \infty} \epsilon_{n}^{k} d_{n} \leq 2 \omega_{k} \inf _{\Omega} \mathcal{M}
$$

In order to prove that

$$
\liminf _{n \rightarrow \infty} \epsilon_{n}^{k} d_{n} \geq 2 \omega_{k} \inf _{\Omega} \mathcal{M}
$$

we use some ideas of [4][Lemma 2.2].

By Lemmas 4.2 and 4.4 it follows that $\tilde{w}_{n}^{i}\left(x^{\prime}, r\right):=\tilde{v}_{n}\left(x^{\prime}, r+P_{n}^{i}\right) \rightarrow \tilde{w}_{i}$ in $C_{l o c}^{2}\left(\mathbb{R}^{N-k}\right)$, where $\tilde{w}_{i} \neq 0$ and satisfies $(4.6)$ with $\left(\bar{y}^{\prime}, \bar{y}^{\prime \prime}\right)=P^{i}$.

For each $R>0$ and up to a subsequence in $n$, Lemma 4.4 with calculations similar to those which have resulted in (4.2) implies that

$$
\begin{aligned}
\epsilon_{n}^{k} I_{n, B_{k}\left(P_{n}^{i}, R\right)}\left(v_{n}\right)= & \omega_{k}\left|P^{i^{\prime \prime}}\right|^{k}\left(\frac{1}{2} \int_{B_{R}(0)}\left(\left|\nabla \tilde{w}_{i}\right|^{2}+V\left(P^{i}\right) \tilde{w}_{i}^{2}\right) d z\right. \\
& \left.-\int_{B_{R}(0)} \tilde{G}\left(r, \tilde{w}_{i}\right) d z\right)+o_{n}(1) \\
\geq & \omega_{k}\left|P^{i^{\prime \prime}}\right|^{k}\left(\frac{1}{2} \int_{B_{R}(0)}\left(\left|\nabla \tilde{w}_{i}\right|^{2}+V\left(P^{i}\right) \tilde{w}_{i}^{2}\right) d z-\int_{B_{R}(0)} F\left(\tilde{w}_{i}\right) d z\right)+o_{n}(1) .
\end{aligned}
$$

Since $\tilde{w}_{i} \in H^{1}\left(\mathbb{R}^{N-k}\right)$, it follows that for a given $\eta>0$ there exists $R>0$ such that

$$
\liminf _{n \rightarrow \infty} \epsilon_{n}^{k} I_{n, B_{k}\left(P_{n}^{i}, R\right)}\left(v_{n}\right) \geq \omega_{k} \mathcal{M}\left(P^{i}\right)-\eta
$$

Taking into account Lemma 4.3, it follows that for $n$ large enough $B_{k}\left(P_{n}^{1}, R\right)$ and $B_{k}\left(P_{n}^{2}, R\right)$ are disjoint, and then

$$
\epsilon_{n}^{k} I_{n}\left(v_{n}\right)=\epsilon_{n}^{k} I_{n, B_{k}\left(P_{n}^{1}, R\right)}\left(v_{n}\right)+\epsilon_{n}^{k} I_{n, B_{k}\left(P_{n}^{2}, R\right)}\left(v_{n}\right)+I_{n, \mathbb{R}^{N} \backslash B_{n, R}}\left(v_{n}\right),
$$

where $B_{n, R}:=B_{k}\left(P_{n}^{1}, R\right) \cup B_{k}\left(P_{n}^{2}, R\right)$. 
Now, a similar calculation to (2.21) in [22] implies that

$$
\liminf _{n \rightarrow \infty} I_{n, \mathbb{R}^{N} \backslash B_{n, R}}\left(v_{n}\right) \geq-\eta
$$

for $R>0$ sufficiently large, and then

$$
\begin{aligned}
\liminf _{n \rightarrow \infty} \epsilon_{n}^{k} I_{n}\left(v_{n}\right) & \geq \omega_{k}\left(\mathcal{M}\left(P^{1}\right)+\mathcal{M}\left(P^{2}\right)\right)-3 \eta \\
& \geq 2 \omega_{k} \inf _{\Omega} \mathcal{M}-3 \eta
\end{aligned}
$$

Since the last inequality holds for all $\eta>0,(4.12)$ is proved.

To prove (ii), let us suppose by contradiction that

$$
\mathcal{M}\left(P^{i}\right)>\inf _{\Omega} \mathcal{M}, \quad i \in\{1,2\}
$$

where $P^{i}$ is given by (4.4). Just by arguing as in the first item, one can see that

$$
\liminf _{n \rightarrow \infty} \epsilon_{n}^{k} I_{n}\left(v_{n}\right) \geq \omega_{k}\left(\mathcal{M}\left(P^{1}\right)+\mathcal{M}\left(P^{2}\right)\right)>2 \omega_{k} \inf _{\Omega} \mathcal{M},
$$

which contradicts the statement of Lemma 4.1. This contradiction proves the proposition.

Remark 4.6 Note that by (ii) of the last result it follows that $P^{i} \in \Omega$. In fact, if $P^{i} \in \partial \Omega$, by $\left(\mathcal{M}_{1}\right), \mathcal{M}\left(P^{i}\right)>\inf _{\Omega} \mathcal{M}$, which together with the continuity of $\mathcal{M}$ (see Lemma 2.1 in [12]) leads to a contradiction.

\section{Proof of Theorem 1.1}

Let $v_{n}$ be as at the beginning of Section 4 and $u_{n}(x):=v_{n}\left(\epsilon_{n}^{-1} x\right)$.

\section{Proposition 5.1}

$$
\lim _{n \rightarrow \infty}\left\|v_{n}\right\|_{L^{\infty}\left(\Omega_{n} \backslash\left(B_{k}\left(P_{n}^{1}, R\right) \cup B_{k}\left(P_{n}^{2}, R\right)\right)\right)}=0 .
$$

Proof The proof follows by contradiction. Let us suppose that there exist $\eta>0$ and sequences $R_{n} \rightarrow \infty$ and $\left(y_{n}\right) \subset \Omega_{n} \backslash\left(B_{k}\left(P_{n}^{1}, R_{n}\right) \cup B_{k}\left(P_{n}^{1}, R_{n}\right)\right)$ such that

$$
\left|v_{n}\left(y_{n}\right)\right| \geq \eta
$$

Since $\epsilon_{n} y_{n} \in \Omega$, it follows that $\epsilon_{n} y_{n} \rightarrow \bar{y} \in \Omega$ up to a subsequence.

Then, following the arguments in Proposition 4.5, it is possible to show that

$$
\epsilon_{n}^{k} d_{n}=I_{n}\left(v_{n}\right) \geq 3 \omega_{k} \inf _{\Omega} \mathcal{M}
$$

which contradicts Lemma 4.1.

By standard elliptic regularity theory, it is possible to show that $v_{n} \in C^{2}\left(\mathbb{R}^{N}\right)$. Then, by continuity, Proposition 5.1 implies that

$$
\left\|v_{n}\right\|_{L^{\infty}\left(\partial\left(B_{k}\left(P_{n}^{1}, R_{n}\right) \cup B_{k}\left(P_{n}^{1}, R_{n}\right)\right)\right)}=o_{n}(1) .
$$


In order to prove the exponential decay with respect to $\epsilon_{n}$ of the functions $u_{\epsilon}$, let us take as a comparison function

$$
W(x)=C\left(e^{-\beta d_{k}\left(x, P_{n}^{1}\right)}+e^{-\beta d_{k}\left(x, P_{n}^{2}\right)}\right)
$$

defined in $\mathbb{R}^{N} \backslash\left(B_{k}\left(P_{n}^{1}, R\right) \cup B_{k}\left(P_{n}^{1}, R\right)\right)$, where $C>0$ is to be chosen. For sufficiently small $\beta>0$ (independent of $\epsilon_{n}$ ), it follows that

$$
\left(-\Delta+V\left(\epsilon_{n} x\right)-\frac{g_{n}\left(\epsilon_{x}, v_{n}\right)}{v_{n}}\right)\left(W \pm v_{n}\right) \geq 0 \quad \text { in } \mathbb{R}^{N} \backslash\left(B_{k}\left(P_{n}^{1}, R\right) \cup B_{k}\left(P_{n}^{1}, R\right)\right)
$$

Then, by (5.1), for $x \in \partial\left(B_{k}\left(P_{n}^{1}, R\right) \cup B_{k}\left(P_{n}^{1}, R\right)\right)$

$$
W(x) \pm v_{n}(x)=C 2 e^{-\beta R} \pm v_{n} \geq 0
$$

for a sufficiently large constant $C>0$ which does not depend on $n$. Hence the maximum principle applies and

$$
\left|v_{n}\right| \leq W(x) \quad \text { on } \mathbb{R}^{N} \backslash\left(B_{k}\left(P_{n}^{1}, R\right) \cup B_{k}\left(P_{n}^{1}, R\right)\right)
$$

Then there exists a sufficiently large constant $C>0$ such that

$$
\left|v_{n}(x)\right| \leq C\left(e^{-\beta d_{k}\left(x, P_{n}^{1}\right)}+e^{-\beta d_{k}\left(x, P_{n}^{2}\right)}\right)
$$

for $x \in \mathbb{R}^{N} \backslash\left(B_{k}\left(P_{n}^{1}, R\right) \cup B_{k}\left(P_{n}^{1}, R\right)\right)$, which implies that

$$
\left|u_{n}(x)\right| \leq C\left(e^{-\frac{\beta}{\epsilon_{n}} d_{k}\left(x, \epsilon_{n} P_{n}^{1}\right)}+e^{-\frac{\beta}{\epsilon_{n}} d_{k}\left(x, \epsilon_{n} P_{n}^{2}\right)}\right)
$$

for $x \in \mathbb{R}^{N} \backslash\left(B_{k}\left(\epsilon_{n} P_{n}^{1}, \epsilon_{n} R\right) \cup B_{k}\left(\epsilon_{n} P_{n}^{1}, \epsilon_{n} R\right)\right)$. Since $P^{1}, P^{2} \in \Omega$, it follows that for large enough $n, \epsilon_{n} P_{n}^{i} \in \Omega$ for $i=1,2$. In particular, by (5.2) it holds that

$$
\left\|u_{n}\right\|_{L^{\infty}\left(\mathbb{R}^{N} \backslash \Omega\right)} \leq C e^{\frac{-\beta}{\epsilon_{n}}}
$$

which implies that $u_{n}$ satisfies the original problem for $n$ large enough. The proof of (1.6) follows by (4.4) and item (ii) of Proposition 4.5. Therefore the proof of Theorem 1.1 follows.

Competing interests

The authors declare that they have no competing interests.

Authors' contributions

All the authors wrote, reviewed and approved the final manuscript.

\section{Author details}

'Faculdade de Matemática, Universidade Federal do Pará, Belém, PA 66075-110, Brazil. ²Departamento de Matemática e Computação, Faculdade de Ciências e Tecnologia, Universidade Estadual Paulista - Unesp, Presidente Prudente, SP 19060-900, Brazil. 


\section{Acknowledgements}

We would like to express our gratitude to Prof. Antonio Suárez for some discussions on this subject and to the anonymous referee for comments and careful reading of the manuscript.

GMF is supported by PROCAD/CASADINHO 552101/2011-7, CNPq/PQ 301242/2011-9 and CNPq/CSF 200237/2012-8. MTOP is supported by FAPESP 2014/16136-1, CNPq 442520/2014-0 and UNESP/PROPe.

Received: 30 March 2015 Accepted: 10 August 2015 Published online: 18 September 2015

\section{References}

1. Floer, A, Weinstein, A: Nonspreading wave packets for the cubic Schrödinger equations with a bounded potential. J. Funct. Anal. 69, 397-408 (1986)

2. Rabinowitz, PH: On a class of nonlinear Schrödinger equations. Z. Angew. Math. Phys. 43, 270-291 (1992)

3. Wang, X: On concentration of positive bound states of nonlinear Schrödinger equations. Comm. Math. Phys. 153 229-244 (1993)

4. del Pino, M, Felmer, P: Local mountain pass for semilinear elliptic problems in unbounded domains. Calc. Var. Partial Differ. Equ. 4, 121-137 (1996)

5. Malchiodi, A, Montenegro, M: Boundary concentration phenomena for a singularly perturbed elliptic problem. Commun. Pure Appl. Math. 55, 1507-1568 (2002)

6. Ambrosetti, A, Malchiodi, A, Ni, W: Singularly perturbed elliptic equations with symmetry: existence of solutions concentrating on spheres, part I. Commun. Math. Phys. 235, 427-466 (2003)

7. Cingolani, S, Pistoia, A: Nonexistence of single blow-up solutions for a nonlinear Schrödinger equation involving critical Sobolev exponent. Z. Angew. Math. Phys. 55, 201-215 (2004)

8. Ambrosetti, A, Malchiodi, A, Ni, W: Singularly perturbed elliptic equations with symmetry: existence of solutions concentrating on spheres, part II. Indiana Univ. Math. J. 53(2), 297-329 (2004)

9. Ruiz, D: Semiclassical states for coupled Schrödinger-Maxwell equations: concentration around a sphere. Math. Models Methods Appl. Sci. 15, 141-164 (2005)

10. D'Aprile, T, Juncheng, W: On bound states concentrating on spheres for the Maxwell-Schrödinger equation. SIAM J. Math. Anal. 37, 321-342 (2005)

11. Ianni, I: Solutions of the Schrödinger-Poisson problem concentrating on spheres, part II: existence. Math. Models Methods Appl. Sci. 19(6), 877-910 (2009)

12. Bonheure, D, Di Cosmo, J, Schaftingen, JV: Nonlinear Schrödinger equation with unbounded or vanishing potentials: solutions concentrating on lower dimensional spheres. J. Differ. Equ. 252, 941-968 (2012)

13. Bonheure, D, Di Cosmo, J, Mercuri, C: Concentration on circles for nonlinear Schrödinger-Poisson systems with unbounded potentials vanishing at infinity. Commun. Contemp. Math. 14(2), 1-30 (2012)

14. Alves, CO, Soares, SHM: On the location and profile of spike-layer nodal solutions to nonlinear Schrödinger equations. J. Math. Anal. Appl. 296, 563-577 (2004)

15. Alves, CO, Soares, SHM: Nodal solutions for singularly perturbed equations with critical exponential growth. J. Differ. Equ. 234, 464-484 (2007)

16. Sato, Y: Sign-changing multi-peak solutions for nonlinear Schrödinger equations with critical frequency. Commun. Pure Appl. Anal. 7(4), 883-903 (2008)

17. Alves, CO, Souto, M: Existence of least energy nodal solution for a Schrödinger-Poisson system in bounded domains Z. Angew. Math. Phys. 65(6), 1153-1166 (2014)

18. Fei, M: Sign-changing multi-peak solutions for nonlinear Schrödinger equations with compactly supported potential Acta Appl. Math. 127, 137-154 (2013)

19. Castro, A, Cossio, J, Neuberger, J: A sign-changing solution for a super linear Dirichlet problem. Rocky Mt. J. Math. 27(4), 1041-1053 (1997)

20. Bartsch, T, Weth, T, Willem, M: Partial symmetry of least energy nodal solutions to some variational problems. J. Anal. Math. 96, 1-18 (2005)

21. Willem, M: Minimax Theorems. Birkhäuser, Boston (1996)

22. Pimenta, M, Soares, S: Existence and concentration of solutions for a class of biharmonic equations. J. Math. Anal. Appl. 390, 274-289 (2012)

\section{Submit your manuscript to a SpringerOpen ${ }^{\circ}$ journal and benefit from:}

$\checkmark$ Convenient online submission

- Rigorous peer review

Immediate publication on acceptance

- Open access: articles freely available online

- High visibility within the field

- Retaining the copyright to your article

Submit your next manuscript at $>$ springeropen.com 